\title{
Variations of Coronal Radiations at Optical Wavelengths
}

\author{
By R I H A RD C. ALT ROCK \\ Phillips Laboratory (AFMC), Geophysics Directorate \\ National Solar Observatory/Sacramento Peak \\ National Optical Astronomy Observatories Sunspot, NM 88349, USA
}

\begin{abstract}
This paper reviews observations of the variation over long and short time scales of solar coronal flux as observed from ground-based optical observatories. This includes data from $\mathrm{Fe} \mathrm{X}, \mathrm{Fe}$ $\mathrm{XIV}, \mathrm{Ca} \mathrm{XV}$ and the white-light electron corona. The various parameters are compared and contrasted as indicators of global coronal structure and output. The overall envelope of $\mathrm{Ca}$ $\mathrm{XV}$ minimizes before and during sunspot minimum, begins its slow rise in phase with the solar cycle, but maximizes late with strong 150-day oscillations resulting in values of zero at the local minima. Within the sensitivity of the observations, the increase of the envelope from Cycle minimum to maximum is over a factor of 30 . Excluding possible secondary maxima and ignoring strong 150-day oscillations, the general envelope of $\mathrm{Fe} \mathrm{X}$ appears to indicate a slow decline in intensity following sunspot maximum. Earlier observations indicate a Cycle minimumto-maximum variation of a factor of 2 to 3 . Later observations indicate a factor of 4 to 5 . The overall picture for Fe XIV indicates that it varies in phase with the sunspot cycle. However, multiple maxima occur. The amplitudes of all observed Cycles are probably approximately equal, and the increase from Cycle minimum to maximum is approximately a factor of 10 . The variation of the polarized and unpolarized white light corona is in phase with the sunspot cycle, although it may have a slower decline to minimum than the sunspot number. The increase from Cycle minimum to maximum is a factor of 2 to 3 . Notable periodicities seen in the emission-line corona are (approximately) $27,150,180,220$ and 340 days and 3.4 years.
\end{abstract}

\section{Introduction}

\subsection{Restrictions}

In the context of this colloquium (The Sun as a Variable Star: Solar and Stellar Irradiance Variations) I will only consider variations in global coronal properties, mainly irradiance. As we will see, coronal "irradiance" in the context of this article may have several different definitions. Another restriction that I will place on this article is to consider only ground-based observations. Although there are space-based observations that could bear on this topic, space limitations will not permit consideration of them. A further restriction will be no consideration of correlations with other global solar parameters. Finally, no discussion of latitudinal variations of the corona will occur.

\subsection{Types of observations}

I will consider variations of the $\mathbf{K}$ (white light) and $\mathbf{L}$ (emission line) coronas (but not the F, or dust, corona). Coronal "proxy" lines, such as He I $1083.0 \mathrm{~nm}$, will not be discussed. Methods of observation considered will include $K$ observations at eclipse or with a coronagraph or "K-Coronameter" (which observes only the component of the $K$ corona that is polarized parallel to the limb) and $L$ observations with a coronagraph (I am unaware of any studies of the temporal variation of the corona utilizing observations of the $L$ corona at eclipse). For clarification, all observations of the optical corona must be made above the limb due to the fact that the signal on the disk would be masked by the "noise" of the disk continuum, which is a factor of $10^{5}$ to $10^{6}$ more intense than the 
signal. Even with this restriction, many types of observations are severely limited by the brightness of the sky near the sun, which often exceeds the brightness of the corona.

Briefly, the $K$ corona is due to Thomson scattering of the disk continuum by electrons in the corona; the radiation is optically thin, and the intensity is proportional to the integral of the electron density along the line of sight. The optical $L$ corona is opticallythin radiation produced by forbidden transitions in highly-ionized species, which are excited by collisions and/or high-energy radiation, and the intensity is proportional to the integral of the electron density along the line of sight raised to a power between 1 and 2 (e.g., Guhathakurta et al. [1992] obtain a power of 1.68 for Fe XIV $530.3 \mathrm{~nm}$ ). Only three lines will be considered in this article, as I am aware of no studies of the temporal variation of other lines. These lines are Fe X $637.4 \mathrm{~nm}$, formed near $1 \mathrm{MK}$; Fe XIV 530.3 $\mathrm{nm}$, formed near $2 \mathrm{MK}$; and $\mathrm{Ca}$ XV $569.4 \mathrm{~nm}$, formed near $3 \mathrm{MK}$. These lines are often referred to as the "red", "green" and "yellow" coronal lines, respectively.

\section{Problem areas}

As might be expected from a discipline whose observations have been made over hundreds of years (the $K$ corona) or for more than 50 years (the $L$ corona), observational techniques and observational programs have varied widely. The types of observations that I will consider have been obtained by spectrographs using film or filters using film, photoelectric sensors or the eye. The observational techniques utilized have been at a non-uniform multitude of heights above the limb, spatial resolutions, latitudes and sensitivities to sky brightness. The accuracy of the definitions of the above parameters has also varied with time and location.

Further problems that bear on attempting to use observations from different locations come from the definitions of the global parameters. A widely-used parameter is one I will generally define as "Limb Flux". This is an average intensity at a given height taken over different coronal features. Definition variations include averages over the entire limb or over restricted latitudes, such as $\pm 60^{\circ}$ or $\pm 30^{\circ}$. Another parameter is "Pseudo-Full-Disk Flux", in which a synoptic map extending from the North pole to the South pole and covering 15 days is projected onto a sphere and then integrated over the visible disk. A variation of that technique is the Slovakian Coronal Index, which includes not only the intensities projected onto the solar disk but also the contribution from the corona above the limb. Obviously the last two techniques only obtain information about what the radio investigators term the "slowly-varying" component of the corona, due to the reliance on observations from 15 consecutive days.

Finally, the observations reported here will use a variety of temporal-averaging windowlengths and methods (e.g., block averages vs. running mean) and may incorporate observations from more than one observatory to form a single parameter.

The single greatest problem area is that of calibration. In order to deduce the temporal variation of coronal radiation, it is necessary to reduce all observations to absolute units. This has proved to be extremely difficult. Although the most-widely used calibration source, the solar disk, may be assumed to be of constant intensity for this purpose, other sources, techniques, step-wedges, neutral-density filters, etc., have evidently been variable and/or themselves poorly calibrated. This will be demonstrated later in a discussion of the intensities obtained by different observatories of the same coronal features at the same time. But suffice it to say that this is a major limitation in the attempt to infer long-term, and even short-term variations of the corona. 


\section{Solar-cycle variations of Ca XV $569.4 \mathrm{~nm}$}

There is only one set of observations that bears on this subject: those obtained at the National Solar Observatory at Sacramento Peak, New Mexico, with the Emission-Line Coronal Photometer (ELCP). This instrument scans a 1.1' aperture around a circle of radius 1.13 solar radii $\left(R_{\odot}\right)$ (for this line), removes the sky background through use of a lockin amplifier and records the intensity every $3^{\circ}$ of latitude. The general character of the observations is that $\mathrm{Ca} \mathrm{XV}$ emission is normally absent except in isolated regions. Sources generally overlie active regions that are or have been flaring. It is not appropriate to study the long-term variation of this line by producing a Pseudo-Full-Disk Flux, since most of the disk will have zero intensity. A better parameter is the maximum value of the emission on each day, averaged over some time interval. This should give a quantitative measure of the average $\mathrm{Ca} \mathrm{XV}$ activity. I have praduced such a parameter and plotted it from the time observations began in 1984 to the present. The 27-day-block-average (i.e., the average over the first 27 days, then the average over days $28-54$, etc.) maximum intensity decreased rapidly to zero through 1984 , fluctuated from zero to low values through 1987, began a slow increase with noise until 1991, when it shot up to a value of 3 millionths of the intensity of disk center at $569.4 \mathrm{~nm}$, oscillated over the next 1.5 years, dropped to zero in mid-1992 and recovered to 1 millionth in late 1992. Considering that sunspot maximum occurred in 1989, it is remarkable that only low levels of activity were observed until 1991. Since major flares maximize a couple of years after sunspot maximum, it seems likely that this parameter is connected with the occurrence of major flare activity. Although only 4 maxima occurred in 1991 and 1992, they were separated by roughly 150 days, which may indicate a connection with the previously-found 153 day period in gamma-ray flares.

We thus see an overall envelope that minimizes before and during sunspot minimum, begins its slow rise in phase with the solar cycle, but maximizes late with strong 150-day oscillations resulting in values of zero at the local minima. Within the sensitivity of these observations, the increase of the envelope from Cycle minimum to maximum is over a factor of 30 .

\section{Solar-cycle variations of Fe X $637.4 \mathrm{~nm}$}

The earliest observations of this line were reported by Trellis (1957) and Waldmeier (1957). Waldmeier reports on Arosa observations of Limb Flux going back to 1940. During the period 1940 to 1952 the data are very noisy. There is a slight indication of a maximum near the time of sunspot maximum in 1947 followed by a slow decline into 1952. There is no indication of a minimum during sunspot minimum in 1944, but there is a major maximum in 1943 preceded by a rise from 1941 through 1942 . The overall variation from a minimum in 1941 through 1943 is a factor of 2 to 3 , and from 1944 to 1947 a factor of 1.5 to 2 .

Trellis (1957) presents Pic du Midi observations from 1943 through 1955. He does not show any indication of the 1943 maximum. He shows smoothed curves representing annual averages and annual maxima of Full-Limb Flux. His curves minimize at or near sunspot minima in 1944 and 1954 and maximize during sunspot maximum in 1947. Each of his curves also shows a secondary maximum in 1950, with a slow decline from then to sunspot minimum. The variation from Cycle minimum to maximum is a factor of 2 in the average values and a factor of 3 in the maximum values.

Rušin al. (1988) report on Lomnický Štít Limb Flux values from 1976 to 1986. They find a maximum near the time of sunspot maximum in early 1980 but also find secondary 
maxima just after sunspot minimum in 1977 and just before sunspot minimum in 1986, the latter thus tending to confirm the results of Waldmeier (1957). There is no indication of a secondary maximum soon after sunspot maximum as found by Trellis (1957). Other than that, the general form of a slow decline towards sunspot minimum confirms Trellis. The range of intensities covered during this epoch is a factor of 4 .

Sacramento Peak ELCP Full-Limb Flux at $1.15 \mathrm{R}_{\odot}$ averaged over 27 days shows yet another story. These values are completely in phase with the solar cycle from the time observations began in 1984 up to the present. The minimum occurred in 1987 and the maximum in 1989. Since then there has been a slow oscillating decline. The nature of these oscillations will be discussed later. The variation from Cycle maximum to minimum is a factor of 5 .

The general nature of the solar cycle variation of $\mathrm{Fe} \mathrm{X}$ remains uncertain if all of these observations are given equal weight. The earlier photographic and visual observations taken 1.04 to $1.06 \mathrm{R}_{\odot}$ above the limb are inconsistent among themselves and with the later photoelectric observations taken higher above the limb. There is an indication of a possibility of secondary maxima at low altitudes at various times, particularly near sunspot minimum. There is almost certainly a maximum near the time of sunspot maximum. Excluding possible secondary maxima and ignoring oscillations, the general envelope appears to indicate a slow decline in intensity following sunspot maximum. Earlier observations indicate a Cycle minimum-to-maximum variation of a factor of 2 to 3. Later observations indicate a factor of 4 to 5 .

\section{Solar-cycle variations of Fe XIV $530.3 \mathrm{~nm}$}

The earliest observations reported by Waldmeier (1957) go back to 1939. In observations similar to those reported for Fe $\mathrm{X}$ he reports very noisy Full-Limb Flux values up through 1943. Interestingly, there is a local maximum in 1943 similar to that reported by him for Fe X. After 1943, the noise level appears to go down, and the intensity rises relatively smoothly from a minimum near sunspot minimum in 1944 to a maximum near sunspot maximum in 1947. The increase from Cycle minimum to maximum is approximately a factor of 4 .

Trellis (1957) reports observations similar to those he reports for Fe X. The Full-Limb Flux values minimize at sunspot minima and maximize at sunspot maximum. Whereas Fe X showed a secondary maximum in 1950 , Fe XIV shows a secondary maximum in 1949 , closer to the major maximum. The larger relative intensity of the secondary maximum in this case combined with the smaller temporal separation from the major maximum gives the first indication of a double maximum in Fe XIV. The increase from Cycle minimum to maximum is approximately a factor of 10 .

Leroy \& Trellis (1974) display annual averages of $\pm 30^{\circ}$-Limb Flux from Pic du Midi, Kislovodsk, Arosa and Norikura during the interval 1957 to 1973. The last three stations were renormalized to match the Pic du Midi scale. The relative variations of the four stations match fairly well from 1959 through approximately 1966. From 1967 through 1969 , Norikura falls significantly below the average of the other three (Arosa and Norikura are reported only through 1969). The overall form is that of maxima one or two years after sunspot maxima in 1958 (there may have been earlier maxima), followed by minima in 1964 or 1965 near sunspot minimum and then broad and/or multiple maxima extending from 1968 through 1972 during Solar Cycle 20. Pic du Midi in particular gives the impression of having two or three maxima, although the intervening minima could be statistical fluctuations (no uncertainty bars are given).

Sýkora (1992) presents a similar analysis covering 1943 through 1990 . He reports semi- 
annual averages of Full-Limb Flux for Pic du Midi, Norikura, Kislovodsk, Lomnický Stít and Sacramento Peak ELCP. Major features of the Pic du Midi curve are double peaks in Cycles 18 and 19 and a triple peak in Cycle 20. Norikura begins in 1951 and shows a double peak in Cycle 19 similar to that of Pic du Midi. After that, the intensity of Norikura drops substantially below the average of the other observatories. It shows single peaks in Cycles 20 and 21. Kislovodsk begins in 1957 and shows the possibility of a double peak in Cycle 19, but only single peaks in Cycles 20 and 21. Lomnický Štít begins in Cycle 20. From 1970 through 1982 it shows a substantially lower intensity than Pic du Midi and Kislovodsk. From then on it tracks Kislovodsk well. Pic du Midi ended observations in 1974, but Sýkora produces a unification of Kislovodsk, Norikura and Pic du Midi before then, based on the Pic du Midi scale, and uses the unified Kislovodsk and Norikura observations after 1973 to extend the Pic du Midi curve. Obviously, this curve is single-peaked in Cycle 21.

Noëns (1990) unifies Pic du Midi and Lomnický Štít observations to obtain a Limb Flux from 1945 through 1985. He also obtains double peaks in Cycle 18 and 19 but further double peaks in Cycles 20 and 21. In 18 and 19 the first peak is higher; they are equal in 20 and reversed in 21.

Rybanský et al. (1993) present values of the Coronal Index (CI), which is similar to a Pseudo-Full-Disk Flux with extensions above the limb. It is based on the intensity scale of Lomnický Śtít. They find a single peak in Cycle 22. The question of single or multiple peaks in cycles 20 and 21 must await a resolution of the reality of a deficit in $C I$ between 1970 and 1982, as first shown by Sýkora (1992).

Ambrož (1993) shows statistically that the Lomnický Štít $C I$ is lower than normal relative to the sunspot number for the first half of Cycle 21 , thus confirming the above speculation.

Rybanský et al. (1994) produce a unification of several stations to obtain the run of $C I$ from 1939 to 1963 . They find a solar-cycle envelope that is dramatically increasing in amplitude from Cycle 18 onwards (observations of Cycle 17 were infrequent), such that Cycle 22 is twice as strong as Cycle 18. This increase is not found in any other data set. For example, Sýkora et al. (1993), using similar data, find no such increase.

Altrock (1990) demonstrated that Sacramento Peak ELCP 27-day averages of FullLimb Flux show four peaks in Cycle 21 of roughly equal amplitude, separated by 1 to 1.5 years. However, if a 13 -month running mean is produced, the four peaks turn into a double peak! In this case, the first peak is the higher. This result opens the possibility that previously-seen double peaks could be an artifact of the averaging technique. The double peak presented by Sýkora (1992) for Lomnický Štít has the second peak higher, strengthening the case that the Lomnický Štít $C I$ is depressed prior to 1982.

Sacramento Peak ELCP 27-day averages of Full-Limb Flux rise by a factor of 10 from minimum in 1976 to the maximum in 1979 and by a factor of 8 from minimum in 1987 to maximum in 1989 , thus coinciding with the sunspot minima and maxima. However, as noted above, there is a distinct departure from the sunspot curve in Cycle 21 and also in Cycle 22. Both Cycles show pulses of activity spaced by 1 to 1.5 years. In these data, this appears to be a constant feature of the Fe XIV corona and must be somehow connected with some basic characteristic of the solar dynamo. The intensity at solar minimum has the same value between Cycles 20 and 21, and 21 and 22. This implies the existence of a constant, basal corona that never disappears.

The overall picture presented by the above investigations indicates that Fe XIV varies in phase with the sunspot cycle. There may have been double peaks in Cycles 18 and 19. However, Sacramento Peak ELCP data indicate that use of a 12 or 13 month running mean, as used in some of the above studies, can mask a greater number of maxima and 
artificially produce double peaks. Cycle 20 was characterized by either a broad maximum or up to 3 peaks. Cycle 21 had either a single maximum (Kislovodsk and Norikura) or 4 maxima (Sacramento Peak). Cycle 22 shows peaks in the Sacramento Peak data similar to Cycle 21, but with a gradually decreasing envelope. The amplitudes of the Cycles are probably approximately equal, in disagreement with the results of Rybanský et al. (1994). In many epochs there were great dissimilarities between observations from different observatories. The increase from Cycle minimum to maximum is approximately a factor of 10 .

\section{Solar-cycle variations of the white-light corona}

Trellis (1957) displays Limb Flux from 1943 through 1955. The average and maximum curves (see Section 4) are in phase, or nearly so, with the sunspot cycle. After maximum, there is a sharp decline of about $10 \%$, followed by a long, monotonic, slow decline until just before minimum, at which time there is a more rapid decline. The increase from minimum to maximum is about a factor of two.

Rušin \& Rybanský (1985) use eclipse observations to obtain total emission of the corona as a function of the phase of the sunspot cycle. They find a curve similar to the sunspot number but with a slight rise or plateau during the last couple of years prior to minimum. There is substantial scatter in their data but also evidence that the total emission is independent of the Cycle number. The increase from minimum to maximum is about a factor of 1.5 .

Hansen et al. (1969) show the rise to the maximum of Cycle 20 from 1964 through 1967 of the polarized Limb Flux (pB) from the High Altitude Observatory (HAO) Mauna Loa Solar Observatory K-Coronameter. Normalized to unity in 1964 , the functional dependences of the Limb Flux at four heights from $1.125 \mathrm{R}_{\odot}$ to $1.75 \mathrm{R}_{\odot}$ are nearly identical and equal to the $10.7 \mathrm{~cm}$ radio-flux dependence. The increase from minimum to maximum is a factor of $\geq 2$, and probably 3 . They also list other historical determinations of factors ranging from 1.8 to 2.5 .

Fisher \& Sime (1984) find that monthly means of $p B$ at $1.5 \mathrm{R}_{\odot}$ are well-correlated with the sunspot number from 1965 through 1983. Although they do not display $p B$ vs. time, one may infer from their Figure 1 that $p B$ increases by a factor of 2 during Cycles 20 and 21 . They also conclude the existence of a basal corona, as was found from the Sacramento Peak ELCP Fe XIV observations above.

Altrock \& Sime (1991) compare Pseudo-Full-Disk Flux values of $p B$ at $1.35 \mathrm{R}_{\odot}$ and Sacramento Peak ELCP Fe XIV at $1.15 \mathrm{R}_{\odot}$. The solar-cycle variation of $p B$ is about a factor of 3 . The maximum phase is long and wandering with isolated outbursts of density fluctuations lasting sometimes only on the order of one rotation. Maxima do not appear to occur at the time of sunspot maximum. Differences between the two data sets are, in part, due to the different density and temperature sensitivities. $p B$ is approximately proportional to the square root of the Fe XIV flux. The short-term activity differences are most probably due to temperature fluctuations.

So we see that the variation of the polarized and unpolarized white light $(K)$ corona is in phase with the sunspot cycle. Although $p B$ appears to be well-correlated with the sunspot number, other observations have indicated some unusual features, including a slower decline to minimum than the sunspot number. $p B$ is approximately proportional to the square root of the Sacramento Peak ELCP Fe XIV Pseudo-Full-Disk Flux. The increase from sunspot minimum to sunspot maximum is a factor of 2 to 3 . 


\section{Higher-frequency periodicities in the $L$ corona}

Altrock et al. (1990) analyzed periodicities in the Sacramento Peak ELCP Full-Limb Flux data from 1973 through 1989. Persistent signals were seen at periods near one year in all three ions. These signals were identified in Fe XIV as being due to surges of activity every 1.0 to 1.5 years during the maximum phase of Cycle 21 . Persistent signals were seen at periods near 7-10 months in Fe XIV and Fe X. Periods in this range have been identified in a number of other data sets. Periods near 155 days were observed in Fe XIV during the maximum phase of Cycle 21 and in Fe X from 1984 onwards. Such oscillations in the Fe X flux are clearly seen over the last two years, a result that was not expected. Possible other periodicities occur near $2.4,2.8,3.4,4.0,4.9,5.8$ and 6.6 years. Periodogram power also appears briefly near 57, 59, 76, 89 and 101 days. No persistent signals were seen at a period of 51 days. Rotational signals are clearly seen; these signals were not investigated.

Rušin \& Zverko (1990) obtained periodicities from $C I$ during Cycles 20 and 21. Persistent periods were 182 and 346 days and $2.2,3.0$ and 5.0 years.

Özgüç (1992) analyzed the multi-station unified data set of Sýkora (1983). He found persistent widespread periods during Cycles $18-20$ at $2.1-2.3,2.5$ and $3.4-3.5$ years. He also found some evidence for periodicity at 15 - 18 months.

Altrock \& Henry (1992) further analyzed periodicities in Sacramento Peak ELCP 27day block averages of Fe X Full-Limb Flux during 1988 to 1992. They found an extremely regular, almost sinusoidal, oscillation of this parameter with a $148 \pm 12$ day period. In the power spectrum this feature was 2.5 times the magnitude of any other period. Figure 1 demonstrates this property.

The analysis of Sacramento Peak ELCP Ca XV data discussed in Section 3 showed a 150-day periodicity during Cycle 22.

Rybanský \& Rušin (1988) and Altrock (1990) have shown extremely regular 27-day rotational fluctuations in Fe XIV during certain epochs; e.g., 1986.

In conclusion, notable periodicities seen in the $L$ corona are (approximately) 27, 150, 180,220 and 340 days and 3.4 years.

\section{Conclusions}

I have reviewed the solar cycle and higher-frequency variations of the emission-line and electron corona. The major conclusions of the study are as follows.

The overall envelope of $\mathrm{Ca} X \mathrm{~V}$ minimizes before and during sunspot minimum, begins its slow rise in phase with the solar cycle, but maximizes late with strong 150-day oscillations resulting in values of zero at the local minima. Within the sensitivity of these observations, the increase of the envelope from Cycle minimum to maximum is over a factor of 30 .

The general nature of the solar cycle variation of $\mathrm{Fe} \mathrm{X}$ remains uncertain if all of the observations are given equal weight. The earlier photographic and visual observations taken 1.04 to $1.06 R_{\odot}$ above the limb are inconsistent among themselves and with the later photoelectric observations taken higher above the limb. There is an indication of a possibility of secondary maxima at low altitudes at various times, particularly near sunspot minimum. There is almost certainly a maximum near the time of sunspot maximum. Excluding possible secondary maxima and ignoring strong 150-day oscillations, the general envelope appears to indicate a slow decline in intensity following sunspot maximum. Earlier observations indicate a minimum-to-maximum variation of a factor of 2 to 3. Later observations indicate a factor of 4 to 5 . 


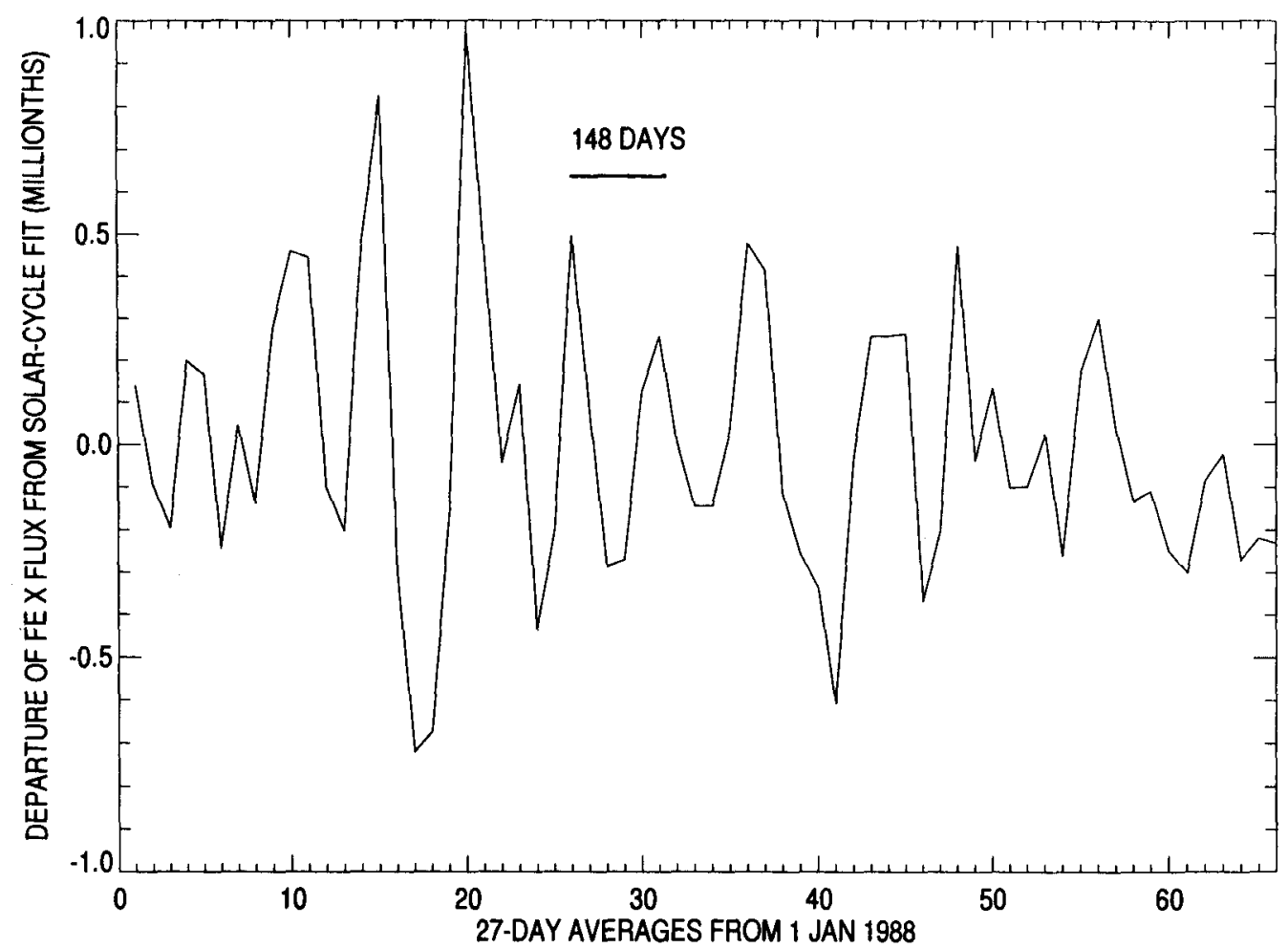

FIgURE 1. Sacramento Peak ELCP 27-day block averages of detrended (solar-cycle variation removed) Fe X Full-Limb Flux from 1988 to 1992 demonstrating a $148 \pm 12$ day period. The values are in millionths of the intensity of disk center at $637.4 \mathrm{~nm}$.

The overall picture for Fe XIV indicates that it varies in phase with the sunspot cycle. There may have been double peaks in Cycles 18 and 19. However, Sacramento Peak $E L C P$ data indicate that use of a 12 or 13 month running mean, as used in some of the studies, can mask a greater number of maxima and artificially produce double peaks. Cycle 20 was characterized by either a broad maximum or up to 3 peaks. Cycle 21 had either a single maximum (Kislovodsk and Norikura) or 4 maxima (Sacramento Peak). Cycle 22 shows similar peaks to Cycle 21 in the Sacramento Peak data, but with a gradually decreasing envelope. The amplitudes of the Cycles are probably approximately equal, in disagreement with the results of Rybanský et al. (1994). In many epochs there were great dissimilarities between observations from different observatories. The increase from Cycle minimum to maximum is approximately a factor of 10 .

H. Hudson (private communication) points out that there is an interesting result in the pattern of Cycle amplitudes. That is, the Fe X, Fe XIV and Ca XV amplitudes are approximately $4-5,10$ and $>30$, respectively. If these variations are scaled to unity at Cycle minimum, the increase in the Cycle-maximum value with ionization temperature consistently implies a hotter corona at solar maximum. Guhathakurta \& Altrock (1992) came to a similar conclusion from the Sacramento Peak ELCP Fe X and Fe XIV data alone, although their study implies that the maximum temperature may occur on the declining phase of the sunspot cycle. They found a minimum temperature of $1.52 \pm$ $0.03 \mathrm{MK}$ during 1988 and maximum temperatures of $1.64 \pm 0.06 \mathrm{MK}$ during 1984 (the first year of joint Fe X and Fe XIV observations) and $1.57 \pm 0.03 \mathrm{MK}$ during 1989 to 
1991. These temperatures are not consistent with the ionization temperature of $\mathrm{Ca} X V$, however, and they either represent a non-flaring component of the corona or the cooler portion of a dual-temperature corona. Altrock \& Sime (1991) found evidence of solarcycle temperature fluctuations through comparison of $K$ and $\mathrm{Fe}$ XIV observations (see Section 6).

The variation of the polarized and unpolarized white light $(K)$ corona is in phase with the sunspot cycle. Although the polarized brightness $(p B)$ appears to be well-correlated with the sunspot number, other observations have indicated some unusual features, including a slower decline to minimum than the sunspot number. $p B$ is approximately proportional to the square root of the Sacramento Peak ELCP Fe XIV Pseudo-Full-Disk Flux. The increase from Cycle minimum to maximum is a factor of 2 to 3 .

Notable periodicities seen in the optical emission-line corona are (approximately) 27, $150,180,220$ and 340 days and 3.4 years.

Acknowledgments. Thanks to Hugh S. Hudson for several helpful comments. I am grateful for support from the U. S. Air Force Office of Scientific Research under Grant PL011. I wish to thank Timothy W. Henry of Sacramento Peak for programming and data reduction and analysis assistance, and Lou B. Gilliam, Chief Observer of the Sacramento Peak John W. Evans Solar Facility for obtaining the observations. The National Optical Astronomy Observatories are operated by the Association of Universities for Research in Astronomy, Inc., (AURA) under cooperative agreement with the National Science Foundation (NSF). The National Solar Observatory is partially supported by the U.S. Air Force under a Memorandum of Understanding with the NSF.

\section{REFERENCES}

Altrock, R.C. 1990 The variation of solar Fe XIV and Fe X flux over 1.5 solar activity cycles. In Climate Impact of Solar Variability (ed. K. H. Schatten \& A. Arking). NASA CP 3086, pp. 287-292.

Altrock, R.C., RADICK, R.R. \& HENRY, T.W. 1990 A search for non-rotational periodicities in solar Fe XIV and Fe X flux during Solar Cycles 21 and 22 (Abstract). Bull. Am. Astron. Soc. 22, 873.

Altrock, R.C. \& Henry, T.W. 1992 Solar Fe XIV and Fe X fluxes as proxies for global solar parameters (Abstract). Trans. Am. Geophys. Union 73(43), Fall Meeting Supplement, 433.

Altrock, R.C. \& SIME, D.G. 1991 Long-term variations in the solar global coronal flux (Abstract). Trans. Am. Geophys. Union 72, Spring Meeting Supplement, 224.

AMBrož, P. 1993 Variability of solar irradiance related to the large-scale convective patterns. Contributed paper presented at IAU Colloquium No. 143 "The Sun as a Variable Star: Solar and Stellar Irradiance Variations, June 20-25, 1993, Boulder, CO.

Fisher, R. \& SimE, D.G. 1984 Solar activity cycle variation of the $K$-corona. Astrophys. J. 285, 354-358.

Guhathakurta, M. \& Altrock, R.C. 1992 The solar cycle variation of coronal temperature during Cycle 22. In The Solar Cycle: Workshop Proceedings, National Solar Observatory/ Sacramento Peak (12th), 15-18 October, 1991 (ed. K.L. Harvey). pp. 395-403. Astronomical Society of the Pacific.

Guhathakurta, M., Rottman, G.J., Fisher, R.R., Orrall, F.Q. \& Altrock, R.C. 1992 Coronal density and temperature structure from coordinated observations associated with the total solar eclipse of 1988 March 18. Astrophys. J. 388, 633-643.

Hansen, R.T., Garcia, C.J., Hansen, S.F. \& Loomis, H.G. 1969 Brightness variations of the white light corona during the years 1964-67. Solar Phys. 7, 417-433.

Leroy, J.L. \& TRELlis, M. 1974 Trois cycles d'activite dans la basse couronne. Astron. Astrophys. 35, 283-288. 
NoËNs, J.-C. 1990 Le cycle d'activite dans la couronne solaire. Astronomie November-December $1990,351-355$.

ÖzGüÇ, A. 1992 Intermediate-term periodicities in the green corona brightness of the Sun. Astrophys. and Space Sci. 187, 197-207.

Rušin, V., RYbanskÝ, M. \& STREČKo, J. 1988 Variations of the red corona over Cycle 21. In Solar and Stellar Coronal Structure and Dynamics: a Festschrift in Honor of Dr. John W. Evans. Proceedings of the Ninth Sacramento Peak Summer Symposium, Sunspot, NM, 17-21 August, 1987, (ed. R.C. Altrock). pp. 288-291. National Solar Observatory.

Rušin, V. \& RYBANSKÝ, M. 1985 Variations of the total brightness of the white-light corona with the phase of the solar cycle. Bull. Astron. Inst. Czechosl. 36, 77-81.

Rušın, V. \& Zverko, J. 1990 Periodicities in the green corona for the Sun as a star. Solar Phys. 128, 261-268.

RYbanský, M., Rušin, V., Gašpar, P. \& Altrock, R.C. 1993 Coronal Index of solar activity VII, years 1988-1991. Solar Phys., submitted.

Rybanský, M., Rušin, V., Minarovjech, M. \& Gašpar, P. 1994 Coronal Index of solar activity: 1939-1963. Solar Physics, in press.

RYBANSKÝ, M. \& RUšIN, V. 1988 Solar $530.3 \mathrm{~nm}$ irradiance variations (1964 - 1986). In Solar and Stellar Coronal Structure and Dynamics: a Festschrift in Honor of Dr. John W. Evans. Proceedings of the Ninth Sacramento Peak Summer Symposium, Sunspot, NM, 17-21 August, 1987. (ed. R.C. Altrock). pp. 342-351. National Solar Observatory.

SÝKoRA, J. 1983 Time-latitude distribution of green corona brightness during solar Cycles 18, 19 and 20. Contrib. Astron. Obs. Skalnate Pleso 11, 25-62.

SÝKorA, J. 1992 Intensity variations of the solar corona over 4.5 solar activity cycles. Contrib. Astron. Obs. Skalnate Pleso 22, 55-67.

SÝkora, J., Bavassano, B., Storini, M. \& PARISI, M. 1993 Long-term variations in the solar corona: a study of latitudinal asymmetries. In Proceedings 1992 STEP Symposium, (ed. $M$. Teague). in press.

Trellis, M. 1957 Contribution a l'etude de la couronne solaire. Ann. D'Ap., Suppl. No. 5.

WALdmeier, M. 1957 Die Sonnenkorona Vol. II, Birkhauser, Basel. 